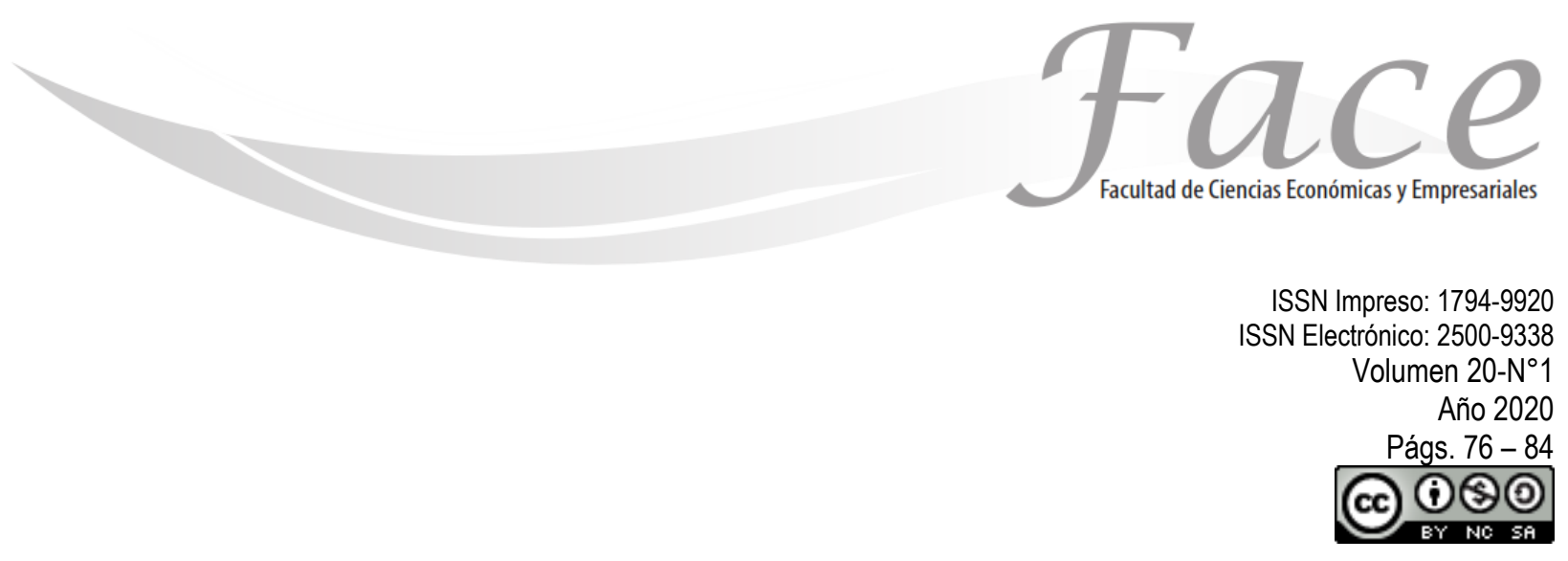

\title{
ANÁLISIS DEL SUSTENTO SOCIAL EN LA PARTICIPACIÓN DE LA POLICIA NACIONAL (PONAL) EN LAS MISIONES DE PAZ DE LA ONU EN EL MARCO DEL POSTCONFLICTO EN COLOMBIA.
}

\author{
Aureliano Sánchez Mejía * \\ https://orcid.org/0000-0001-6398-1975 \\ Steven Aldemar Mora Garzón ** \\ https://orcid.org/0000-0003-2418-7140 \\ Ernesto Fajardo Pascagaza *** \\ https://orcid.org/0000-0003-1168-9512
}

Fecha de Recepción: Marzo 17 de 2020

Fecha de Aprobación: Junio 30 de 2020

\section{Resumen:}

El propósito de esta investigación es analizar la importancia que tiene Colombia para la participación de la Policía Nacional en la misión de paz de la ONU, teniendo en su conjunto su experiencia (táctica, estratégica y humanística) y su impacto social en un proyecto. paz duradera para la nación en el marco del posconflicto. Se implementó una metodología cualitativa, delineando las bases sociales de la investigación. Se aplicó una entrevista semiestructurada para obtener información sobre las capacidades tácticas, estratégicas y humanísticas adquiridas, acentuadas y fortalecidas por el PONAL como resultado de su participación activa y constante en misiones. El aporte investigativo asume la experiencia social adquirida por la policía al interactuar en conflictos armados externos y su mitigación a través de protocolos para el restablecimiento y prolongación de la paz en los territorios; esta experiencia ofrece apoyo y apoyo social a la participación de los miembros de la Policía en la misión de paz de la ONU, mostrando la importancia de adquirir habilidades y contenidos pragmáticos adecuados, para contribuir a la institución a través del servicio policial en las diferentes etapas del policía. posconflicto en Colombia y la consolidación de una paz estable y duradera.

Palabras clave: Misiones de Paz, Postconflicto, Servicio Policial, Sustento Social, ONU. 


\section{ANALYSIS OF SOCIAL SUPPORT IN UN PEACE MISSIONS IN THE FRAMEWORK OF THE POST-CONFLICT IN PARTICIPATION OF THE NATIONAL POLICE (PONAL) IN COLOMBIA.Abstract:}

The purpose of this investigation is to analyze the importance that Colombia has for the participation of the National Police in the UN peacekeeping mission, having as a whole its experience (tactical, strategic and humanistic) and its social impact on a project. lasting peace for the nation in the postconflict framework. A qualitative methodology was implemented, outlining the social bases of research. A semi-structured interview was applied to obtain information on the acquired tactical, strategic and humanistic capacities, accentuated and strengthened by the PONAL as a result of his active and constant participation in missions. The investigative contribution assumes the social experience acquired by the police while interacting in external armed conflicts and their mitigation through protocols for the restoration and prolongation of peace in the territories; this experience offers support and social support to the participation of the members of the Police in the UN peace mission, showing the importance of acquiring skills and suitable pragmatic content, in order to contribute to the institution through the police service in the different stages of the police. postconflict in Colombia and the consolidation of a stable and lasting peace.

Keywords: Missions of Peace, Post-conflict, Police Service, Social Support, UN.

\section{ANÁLISE DO APOIO SOCIAL NAS MISSÕES DA ONU DE PAZ NO ÂMBITO DO PÓS-CONFLITO NA PARTICIPAÇÃO DA POLÍCIA NACIONAL (PONAL) NA COLÔMBIA.}

\section{Resumo:}

O objetivo desta investigação é analisar a importância que a Colômbia tem para a participação da Polícia Nacional na missão de paz da ONU, tendo como um todo sua experiência (tática, estratégica e humanística) e seu impacto social em um projeto. paz duradoura para a nação na estrutura pós-conflito. Uma metodologia qualitativa foi implementada, delineando as bases sociais da pesquisa. Foi aplicada uma entrevista semiestruturada para obter informações sobre as capacidades táticas, estratégicas e humanísticas adquiridas, acentuadas e reforçadas pelo PONAL em resultado da sua participação ativa e constante nas missões. A contribuição investigativa pressupõe a experiência social adquirida pela polícia na interação em conflitos armados externos e sua mitigação por meio de protocolos de restauração e prolongamento da paz nos territórios; esta experiência oferece suporte e apoio social à participação dos membros da Polícia na missão de paz da ONU, mostrando a importância da aquisição de competências e conteúdos pragmáticos adequados, de forma a contribuir com a instituição através do serviço policial nas diferentes fases do polícia. pós-conflito na Colômbia e a consolidação de uma paz estável e duradoura.

Palavras-chave: Missões de Paz, Pós-conflito, Serviço Policial, Apoio Social, ONU. 


\section{INTRODUCCIÓN:}

Es prioridad para el País encontrar herramientas que fortalezcan la proyección internacional, y una de estas herramientas es la participación de la Policía Nacional en las Misiones del Mantenimiento de la Paz de la ONU. Este trabajo busca encontrar y responder la pregunta ¿Cuáles son los beneficios sociales que recibe Colombia al mantener una participación activa de la Policía nacional en las Misiones del Mantenimiento de la Paz de la ONU?, ya que en una óptica primaria se evidencia la problemática de la no coherencia en el móvil del envío de efectivos de la Policía a las Misiones de Paz, cuando precisamente Colombia es un país que cumple con los requisitos de país receptor y no de emisor. Es aquí donde nace la necesidad de que exista un documento académico y científico mediante el cual se ubiquen los beneficios y argumentos Sociales en el marco del postconflicto que sustenten él envió de personal de la Policía Nacional a las Misiones del Mantenimiento de la Paz de la ONU.

Las Misiones para el Mantenimiento de la Paz constituyen uno de los instrumentos con que cuenta la Organización de las Naciones Unidas (ONU) para dar cumplimiento a su cometido de mantener la seguridad y la paz internacional al "prevenir y eliminar amenazas a la paz, y para suprimir actos de agresión u otros quebrantamientos de la paz; y lograr por medios pacíficos, y de conformidad con los principios de la justicia y de derecho internacional," (Art. 1, Carta de las Naciones Unidas, s. f.). Dichos componentes son esenciales para el desarrollo economico del pais mediante la destinacion y fortalecimiento de recursos tanto economicos como talento humano que logren la creacion y el desarrollo de una cadena productiva solida y eficiente (Lombana, 2018).

La policía colombiana es un actor activo desde 1992 en la misiones de paz de la Naciones Unidas mediante su participación en distintas regiones del planeta donde se ha requerido la intervención de la ONU, tales como: "Camboya (1992 con el APRONUC), Yugoslavia (1992 UNPROFOR), El Salvador (1993 ONUSAL), Guatemala (1997 MINUGUA), Haití (2006-2017 MINUSTH), Guinea-Bissau (2009-2016 UNIOGBIS) y Sierra Leona (2000 UNIPSIL)" (Policía Nacional, 2010, pp.38-39).
Nuestros contingentes policiales han brindado apoyo en la reforma de la policía de Haití y en el mantenimiento de la seguridad de su territorio. Igualmente en Guinea Bissau apoyo tanto la reforma de la policía como en el mantenimiento de la seguridad y adicional a ello se apalancarón proyectos para la seguridad integral del país a través de mecanismos del servicio policial, militar y de justicia. De igual manera su labor siempre ha estado enfocada en la protección de los derechos humanos de la población en todos los países que ha intervenido.

Dicha participación ha venido constituyendo para la institución una amplia experiencia en todo tipo de conflictos y situaciones de carácter humanitario, brindando así un incremento en sus capacidades sociales y humanísticas en pro de la comunidad, lo cual permite establecer que esta experiencia es un valor agregado con las características adecuadas y capases de fortalecer y aportar en gran medida en el desarrollo humano y social de las comunidades de nuestro territorio nacional. Donde el estado colombiano a través de su capacidad de articulación entre "el sector público y privado, paralelo a los diálogos de paz debe trabajar en desarrollar estrategias que permitan adelantar acciones claras y efectivas para enfrentar los problemas de empleo, inseguridad y violencia que se viven actualmente Colombia" (Rodríguez, L. A. J \& Suárez, R. G, 2017).

Génesis de las misiones de paz de la ONU.

En la búsqueda del sustento social de la participación de la Policía Nacional en las misiones para el Mantenimiento de la Paz de la ONU, es preciso enmarcar las bases teóricas que soporten los postulados de este documento con el designio de consagrar a la "investigación un sistema coordinado y coherente (...) Es decir, tratar de integrar al problema dentro de un ámbito donde éste cobre sentido, incorporando los conocimientos previos relativos al mismo y ordenándolos de modo tal que resulten útil a nuestra tarea" (Sabino, 1996, p.48), es por ello que se abordara a través del postulado de la génesis de las misiones para el mantenimiento de la paz de la ONU, las cual el secretario general de la Organización Naciones Unidas para 1993, reseña en dos etapas históricas de las operaciones para el mantenimiento 
de la paz, una de ellas fueron las misiones desarrolladas durante la Guerra Fría y la otra son las misiones posteriores (Boutros-Ghali, 1993, p.1).

\section{Evolución de las misiones de Paz de la ONU}

Los postulados generacionales permiten evidenciar que el horizonte de las Misiones para el Mantenimiento de la Paz de la ONU ha venido evolucionando a través de la historia, hasta llegar al suceso cuando "surgieron conceptos como la diplomacia preventiva y el establecimiento de la paz, el mantenimiento de la paz, la consolidación de la paz y, como última ratio, la imposición de la paz" (Vallejo Almeida, 2015, p39).

Partiendo del objetivo primordial de la ONU y bajo la concideración de que su visión se enmarca en la búsqueda continua de mantener la paz internacional, es fundamental que sus misiones consagren el respeto por los derechos primordiales de todas las personas a nivel internacional, sin exepción de raza, genero 0 cualquier otra diferenciación, puesto que su función debe ir más allá del deber ser de lo estipulado en la carta de las naciones unidas y propender en lograr la "solución de problemas internacionales de carácter económico, social, cultural o humanitario, en el desarrollo y estímulo del respeto a los derechos humanos y a las libertades fundamentales (...), sin hacer distinción por motivos de raza, sexo, idioma 0 religión" (ONU, s.f., art. 1, num. 3).

Es así como las Misiones para el Mantenimiento de la Paz de la ONU pueden llegar a fomentar e incentivar en las poblaciones el bienestar y el desarrollo humano como requisito de paz, mediante la prevención de conflictos, el establecimiento de las condiciones de paz, el mantenimiento de la paz, la Aplicación (coercitiva) de la paz y por último la Consolidación de la paz. (ONU, 2008, p.11-12).

\section{La Policía de las Naciones Unidas}

Fue a partir de 1960 que las Naciones Unidas dio comienzo a la utilización de cuerpos policiales en las Misiones para el Mantenimiento de la Paz, cuando por medio de la resolución 143 del Consejo de Seguridad se autoriza disponer un contingente de 30 policías para apoyar las operaciones de las Naciones Unidas en el Congo, después de esto, la policía de las Naciones Unidas ha venido incrementando el número de sus uniformados pasando de 1.667 en 1994 a 14.333 en 2011, logrando tener presencia en 6 misiones políticas especiales y en 12 misiones de mantenimiento de la paz.

Las funciones delegadas a la policía se ha venido incrementando a través del tiempo, puesto que durante las primeras misiones los efectivos cumplían principalmente un roll de observadores, función que hoy día se ha expandido a la participación directa en las operaciones de paz mediante la reconstrucción de fuerzas de policía de los países receptores de las misiones.

Las Misiones para el Mantenimiento de la Paz de la ONU, busca restablecer el orden y la autoridad en los lugares donde por diferentes circunstancias agentes no estatales aprovechan para vulnerar los derechos fundamentales de la población, debido a la ausencia de la autoridad legítima, es por ello que la policía de las Naciones Unidas está llamada a restablecer la paz mediante su función de gobernanza, prevención, descubrimiento e indagación de transgresiones; salvaguardar personas y propiedad; y el sostenimiento del orden público y la seguridad.

La Organización de las Naciones Unidas fundamenta que la "Policía (...) tiene la obligación de respetar y proteger los derechos humanos. Esta interpretación (...) debe ser el denominador común para los agentes de policía de las Naciones Unidas (...) con diversos marcos jurídicos" (ONU, 2016, p.3).

Colombia en las misiones de paz de la ONU.

Las Participación activa en las Misiones del Mantenimiento de la Paz de la ONU es el resultado de la puesta en marcha de una política exterior concreta con intereses definidos por los países participantes. Colombia, como país participante, fundamenta su participación en las misiones de paz bajo el interés de contribuir al mantenimiento de la paz internacional y nacional como instrumento de política pública. Esto es especialmente sensible cuando se refiere a la política exterior, entendida como un reflejo de la situación interna y como un subproducto de la capacidad de un Estado para organizar voluntades y esfuerzos en el exterior a favor de sus propios intereses.

Si se entiende de manera amplia la política de defensa y seguridad nacional de Colombia como una política pública, se logra revelar su objetivo de posicionar a Colombia como un líder en temas de la defensa y la seguridad; primero en el ámbito hemisférico, y 
posteriormente en el global, lo cual se fundamenta en la tesis de que los Estados soberanos consiguen manifestarse como optimizadores de las utilidades en la consecución de sus objetivos particulares y que adicional a ello están atentos a mantener su ventaja ante otros estados, debido a que se ven cohesionados a maniobrar en un entorno de anarquía en el cual su bienestar y seguridad obedecen a su capacidad de activar y mover sus recursos propios (Krasner, 1999, p. 32).

Pareciera utópico este planteamiento para Colombia, pero es su participación activa en las actividades de las Misiones de Paz de la ONU lo que le ha brindado la oportunidad al país de destacarse como un estado que cuenta con altas capacidades tácticas y operacionales en el interior de sus fuerzas armadas y de policía.

De igual manera el estado colombiano ha desempeñado un papel importante en las Misiones de paz de la ONU, debido a que sus fuerzas militares y de policía han adquirido una experiencia invaluable como respuesta al conflicto armado interno y su lucha contra el terrorismo y el narcotráfico. En este sentido se ratifica que "Tanto el gobierno como los observadores militares conceptúan que esta participación internacional permite una beneficiosa transferencia de conocimientos y la posibilidad de recibir cooperación por reciprocidad en caso de ser necesaria" (Pastrana, 2019, p.358), lo cual es de suma importancia en el marco de post acuerdo de paz.

Por otra parte también se expone la necesidad de examinar las competencias (educativas y profesionales) de los uniformados de la policía nacional que son enviados a las misiones de paz de la ONU, para que estos puedan tener un mejor desempeño y ocupen cargos de mayor liderazgo, lo cual es posible "Mediante la construcción de una matriz de diagnóstico del perfil ocupacional requerido por Naciones Unidas, en relación con las capacidades del oficial de la policía colombiana" (Alvarez, 2019, p.86).

Teniendo en cuento todo lo anterior se hace indispensable establecer cuan importante ha sido la participación de la Policía Nacional de Colombia después de 28 años en la Misiones de Mantenimiento de Paz de la ONU, tanto en el aporte como en la adquisición de conocimiento en materia táctica, opercional, humanística y social, como base fundamental para soportar socialmente en el marco del postconflicto la continuidad y fortalecimiento de la participación de la PONAL en las misiones de paz de la ONU.

\section{METODOLOGÍA:}

\subsection{Diseño}

Partiendo de que, uno de los objetivos primordiales de los estudios científicos y sociales es el lograr describir los acontecimientos y sus circunstancias de la manera más fiel y precisa posible teniendo en cuenta sus causales (Babbie, 2000, p.74), se hizo necesario llevar a cabo esta investigación mediante la realización de un vasto trabajo de recopilación documental como insumo primordial para la interpretación del sustento social de la participación de la Policía Nacional en las Misiones de paz de la ONU en el marco del postconflicto en Colombia.

El tipo de estudio que se estableció para esta investigación es de carácter cualitativo, puesto que este prototipo investigativo se fundamenta en sistemáticas de recaudación de datos carentes de cálculo numérico, tales como las expresiones descriptivas y procesos de observación. Su propósito es asentar una realidad de manera estrecha teniendo en cuenta los distintivos del mismo y sus interacciones con el evento que se analiza.

\subsection{Instrumentos}

Es indispensable aclarar que esta investigación es de tipo documental, ya que posee la peculiaridad de manejar como fuente principal de insumos distintos documentos escritos y visuales tales como: documentos impresos, audiovisuales o electrónicos (Morales, 2016, p.2). Sin embargo, también se recurrió a la investigación de campo mediante la realización de una encuesta semiestructurada a los uniformados activos de la PONAL que han sido miembros de las misiones de paz de la ONU, debido a que "se pueden recurrir a otras fuentes distintas a las bibliográficas como, por ejemplo, el testimonio de los hechos, de testigos calificados" (Kaufman \& Rodríguez, 1993, p.43).

Durante el proceso de esta investigación se utilizaron varios métodos: el inductivo y deductivo, al igual que el sintético y analítico. El método inductivo y deductivo se 
utilizó mediante la realización de encuestas a los uniformados activos de la Policía Nacional que han sido miembros de las misiones de paz de la ONU, permitiendo de esta manera poder homogenizar el criterio de los mismos frente a los resultados obtenidos y la importancia de dichas misiones en términos sociales. El método analítico y sintético permitió la realización de una revisión ordenada de la información y los elementos estudiados, al igual que la unificación y relación de hechos aislados aparentemente, que en realidad poseen la capacidad de brindar elementos concluyentes de la importancia de las misiones de paz de la ONU.

Para la realización de esta investigación se logró disponer de igual manera tanto de datos primarios como de secundarios gracias a la utilización de distintas técnicas para su recolección y el acceso a los mismos a través del análisis de documentos históricos, archivos y bibliografía existente del tema estudiado. De igual manera, se utilizó como técnica directa la realización de encuestas personales a través de llamadas telefónicas y correos electrónicos a los uniformados activos de la PONAL que han sido miembros de las misiones de paz de la ONU.

\subsection{Población y muestra}

Para la realización de la encuesta semiestructurada, se estipuló en primera instancia una muestra estadística mediante la utilización de un muestreo probabilístico, en la cual se percató un margen de error del $5 \%$ con un nivel de confianza del $95 \%$ como se establece en la siguiente ecuación:

\section{Ecuación 1.}

Ecuación para muestra probabilística.

$\mathrm{n}=\frac{N * Z^{2} * p * q}{\sigma^{2}(N-1)+Z^{2} * p * q}$

Tabla 1.

Abreviatura estadística de la muestra
Cálculos propios a partir de información estadística necesaria para muestreo.

Teniendo en cuenta la información anterior se partió de un universo estadístico de 847 uniformados de la PONAL que han participado históricamente de las misiones de paz de la ONU, luego se estableció como población objetivo a los uniformados activos que han participado de dichas misiones, es decir 70 policías, lo cual generó como resultado una muestra de 59 encuestas. Apelando a que los resultados de la muestra están muy cercanos a la totalidad de la población objetivo, se determinó en segunda instancia la realización de la encuesta a la totalidad de la población objetivo, es decir a los 70 uniformados activos que han participado en las misiones de paz de la ONU para lograr un $100 \%$ en el nivel de confianza de los resultados obtenidos en esta investigación.

\subsection{Análisis de los datos}

Partiendo de la información recopilada y generada en el transcurso de esta investigación, se realizaron ejercicios descriptivos y explicativos teniendo en cuenta los acontecimientos históricos y actuales de tipo estructural. De igual manera toda la información recopilada ha sido objeto de convalidación mediante el análisis semántico de categorías cualitativas y sus datos ha sido procesados mediante el software Microsoft Excel para el establecimiento visual de tablas y gráficas, las cuales son esenciales para el análisis e interpretación de los resultados con el propósito de fundamentar el sustento social de la participación de la Policía Nacional en las Misiones de paz de la ONU y su importancia en el marco del postconflicto en Colombia.

\begin{tabular}{|c|c|}
\hline Abreviatura/símbolo & Definición \\
\hline N & Muestra \\
\hline N & Población \\
\hline Z & Nivel de confianza $(1,96)$ \\
\hline$P$ & Probabilidad de éxito $(0,5)$ \\
\hline Q & Probabilidad de Fracaso $(0,5)$ \\
\hline$\sigma$ & Error $(0,05)$ \\
\hline
\end{tabular}




\section{RESULTADOS:}

Durante el trascurso de esta investigación se estudiaron los componentes tácticos, estratégicos, sociales y humanísticos con el propósito de descubrir el nivel de capacidades que adquiere y aporta la policía nacional mediante su participación en las misiones de paz de la ONU, de igual manera se analizó el nivel de conocimiento adquirido de algunos componentes sociales que son esenciales en la labor policial en el marco del postconflicto.

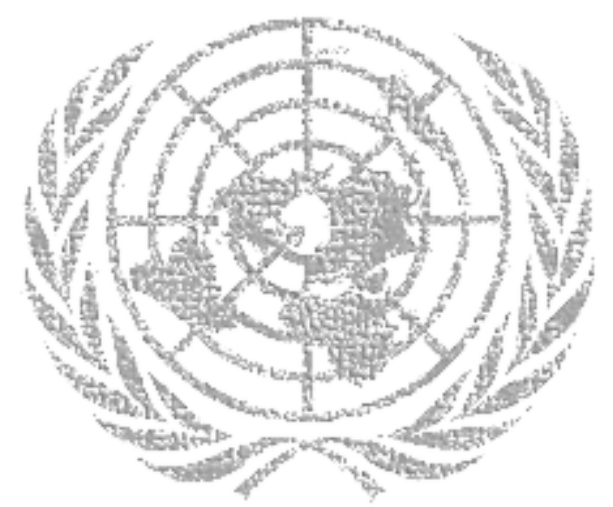

Figura 1.

Nivel de Capacidades y conocimiento aportado V/S adquirido en misiones de paz de la ONU.
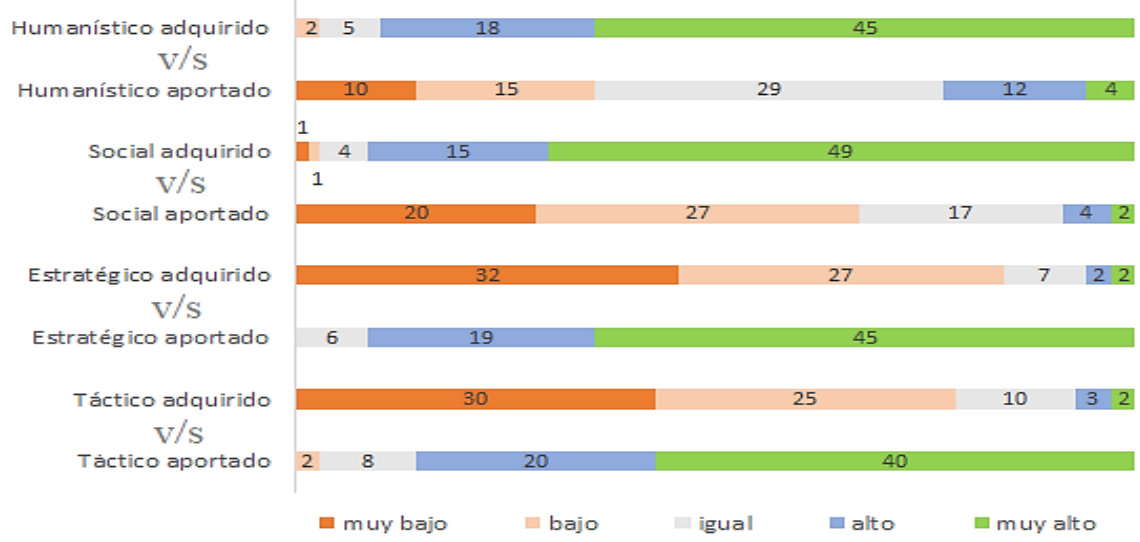

Fuente: Cálculos propios a partir de información de encuesta.

\section{Figura 2.}

Nivel de capacidades y conocimiento social adquirido en las misiones de paz de la ONU.

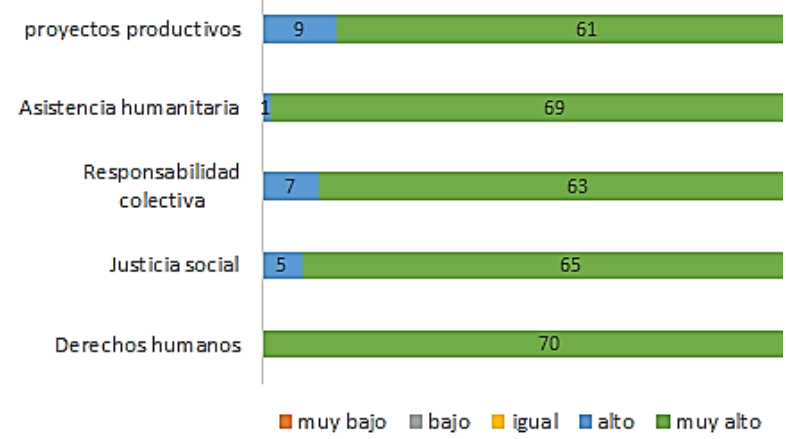

Fuente: Cálculos propios a partir de información de encuesta. 
A partir de la figura 2, es posible determinar el altísimo impacto positivo en el componente social que genera en los uniformados de la PONAL su participación en las misiones de paz de la ONU, puesto que el $100 \%$ de los encuestados (70 uniformados) afirman que los niveles de conocimiento adquiridos en los módulos analizados son de alta y muy alta representación durante el ejercicio policial en las misiones $y$ operaciones de paz.

Cabe destacar con respecto a los resultados, que el módulo de los derechos humanos establece la mayor importancia para las misiones de paz de la ONU, puesto que presenta un nivel muy alto en el grado de adquisición de conocimiento para el $100 \%$ de los uniformados de la PONAL.

\section{DISCUSIÓN DE RESULTADOS:}

Al realizar un contraste bibliográfico y experimental del porqué de la presencia y participación de la Policía Nacional de Colombia en las misiones de paz de la ONU, es de suma importancia determinar si la experiencia adquirida en el recorrido histórico es suficiente para establecer la necesidad de continuar siendo participe de dichas misiones. Es allí donde toma relevancia la derivación de esta investigación, puesto que los resultados obtenidos bajo el fundamento social logran construir una articulación científica que soporta la existencia de una gran cantidad de beneficios sociales que recibe Colombia, al mantener una participación activa de la Policía nacional en las Misiones del Mantenimiento de la Paz de la ONU teniendo en cuenta la trasferencia reciproca de conocimiento, donde la PONAL aporta a través de sus uniformados altos niveles de conocimiento tactito $y$ operacional y en contravención recibe altos niveles sociales y humanísticos para los mismos, destacándose en gran medida los módulos de derechos humanos, asistencia humanitaria y justicia social, los cuales son esenciales en todas las actividades que pueda llevar a cabo la policía nacional como institución encargada de salvaguardar las condiciones necesarias para el ejercicio de los derechos y libertades públicas de los excombatientes en el marco del postconflicto en Colombia.

\section{CONCLUSIONES:}

Es claro que la policial nacional de Colombia ha logrado incrementar y fortalecer las capacidades y el conocimiento en los componentes sociales y humanísticos como resultado de su participación histórica en las misiones de paz de la ONU, lo cual le brinda herramientas adecuadas para intervenir de manera apropiada en las diferentes fases del postconflicto cuando así sea requerido.

Teniendo en cuenta la existencia de una trasferencia de conocimiento reciproca con la ONU, se hace imperante fortalecer y mantener en el tiempo la participación activa de la PONAL en las misiones de paz de la ONU. Esto con el propósito de conservar lasos multilaterales en la lucha trasnacional de la criminalidad en todos sus ámbitos.

\section{REFERENCIAS:}

Alvarez, C. A. (2019). Análisis de la Cooperación y Participación de la Policía Nacional en Operaciones de Mantenimiento de la Paz ante las Naciones Unidas. Bogotá: Escuela de Posgrados de Policía Nacional.

Babbie, E. (2000). Fundamentos de la Investigación Social. México, D.F: Internacional Thompson.

Boutros-Ghali, B. (1993). Maintaining International Peace and Security: Los Angeles, EE.UU: Organización de Naciones Unidas. 
Kaufman, A. M., \& Rodríguez , M. E. (1993). La escuela $y$ los textos. Buenos Aires, Argentina: Santillana.

Krasner, S. (1999). Sovereignty: Organized Hypocrisy. New Jesey: Universidad de Princeton.

Lombana, J, Amashta, Y, Correa, C, \& Rodríguez. (2018). Benchmarking y análisis de competitividad de las cadenas productivas de quinua en Colombia, Perú y Bolivia. FACE: Revista de la Facultad de Ciencias Económicas y Empresariales, 17 (2), 157-16. En:

http://revistas.unipamplona.edu.co/ojs_vicein ves/index.php/FACE/article/view/2891

Morales, O. (2016). Fundamentos de la Investigación Documental. Mérida, Venezuela: Universidad de los Andes (ULA).

ONU. (2008). United Nations Peacekeeping Operations Principles and Guidelines. New York, NY: Naciones Unidas.

ONU. (2016). Informe del Secretario General sobre la labor policial de las Naciones Unidas. New York: Naciones Unidas (Consejo de Seguridad).

Organización de las Naciones Unidas. (s.f.). Carta de las Naciones Unidas. Obtenido de https://www.un.org/es/sections/uncharter/chapter-i/index.html

Organización de las Naciones Unidas. (s.f.). Carta de las Naciones Unidas: Articulo 1. New York: ONU.

Pastrana, E. (2019). Fuerzas Militares de Colombia: nuevos roles y desafíos nacionales e internacionales. Bogotá: Hubert Gehring Editores.

Policía Nacional. (2010). Manual de Cooperación Internacional de la Policía Nacional de Colombia, Tomo 2. Bogotá: PONAL.
Rodríguez, L. A. J, \& Suárez, R. G. (2017). Gestión del Post-conflicto como estrategia de la Responsabilidad Social en las empresas. FACE: Revista de la Facultad de Ciencias Económicas y Empresariales, 17(1), 35-43. En:

http://revistas.unipamplona.edu.co/ojs_vicein ves/index.php/FACE/article/view/2576

Sabino, C. (1996). El proceso de investigación. Buenos Aires, Argentina: Lumen-Humanitas.

Vallejo Almeida, G. (2015). Las operaciones de paz de la ONU, una opción para el caso colombiano. JUS público, 39. 